American Journal of Economics and Business Administration 3 (1): 186-190, 2011

ISSN 1945-5488

(C) 2010 Science Publications

\title{
Automating Commercial Video Game Development using Computational Intelligence
}

\author{
${ }^{1}$ Tse Guan Tan, ${ }^{1}$ Jason Teo and ${ }^{2}$ Patricia Anthony \\ ${ }^{1}$ Evolutionary Computing Laboratory, School of Engineering and Information Technology, \\ Universiti Malaysia Sabah, Malaysia \\ ${ }^{2}$ Center of Excellence in Semantic Agents, Universiti Malaysia Sabah, Malaysia
}

\begin{abstract}
Problem statement: The retail sales of computer and video games have grown enormously during the last few years, not just in United States (US), but also all over the world. This is the reason a lot of game developers and academic researchers have focused on game related technologies, such as graphics, audio, physics and Artificial Intelligence (AI) with the goal of creating newer and more fun games. In recent years, there has been an increasing interest in game AI for producing intelligent game objects and characters that can carry out their tasks autonomously. Approach: The aim of this study is an attempt to create an autonomous intelligent controller to play the game with no human intervention. Our approach is to use a simple but powerful evolutionary algorithm called Evolution Strategies (ES) to evolve the connection weights and biases of feed-forward Artificial Neural Networks (ANN) and to examine its learning ability through computational experiments in a non-deterministic and dynamic environment, which is the well-known arcade game, called Ms. Pac-man. The resulting algorithm is referred to as an Evolution Strategies Neural Network or ESNet. Results: The comparison of ESNet with two random systems, Random Direction (RandDir) and Random Neural Network (RandNet) yields promising results. The contribution of this work also focused on the comparison between the ESNet with different mutation probabilities. The results show that ESNet with a high probability with high mean scores recorded compared to the mean scores of RandDir, RandNet and ESNet with a low probability. Conclusion: Overall, the proposed algorithm has a very good performance with a high probability of automatically generating successful game AI controllers for the video game.
\end{abstract}

Key words: Artificial Neural Networks (ANN), Ms. Pac-man, computational intelligence, Evolution Strategies (ES), Non-Player Characters (NPCs), Artificial Intelligence and Interactive Digital Entertainment (AIIDE), Congress on Evolutionary Computation (CEC), mutation probability, random direction

\section{INTRODUCTION}

The computer and video game industry is currently one of the most attractive and profitable industries in the world. This industry is now arguably larger than the entertainment industries, such as film and music (Jayakanthan, 2002) and it has huge potential to dominate the art form of the 21st century. Table 1 presents the NPD sales figures for the US computer and video game industry between year 2002 and year 2009. NPD (NPD, 1967) is a world-leading provider of consumer and retail market research information. According to NPD's annual reports 2002-2005, the four-year average US retail sales of computer and video games was $\$ 11.34$ billion ( $\mathrm{S} \mathrm{D}=0.30$ ). Both of these games have remained essentially unchanged from 2002-
2005, which are $\$ 11.70$ billion in 2002 (Riley, 2004), $\$ 11.20$ billion in 2003 (Riley, 2004), $\$ 11.00$ billion in 2004 (Riley, 2005) and \$11.45 billion in 2005 (Riley, 2006). But, the total sales have significantly increased over the next 3 years from 2006-2008. In 2006, the market for computer and video games in US is growing, with sales of $\$ 13.47$ billion (Riley, 2007) marking a $18 \%$ increase over the previous record achieved in 2005. Furthermore, the total industry experienced the greatest percentage growth rates, with a significant $40 \%$ rise in 2007 when benchmarked to 2006, generated revenues exceeding $\$ 18.8$ billion (Riley, 2008). In annual 2008, the retail sales reached $\$ 22.10$ billion (Riley, 2010) and achieved highest sales over the previous records, a 17 percent increase over the $\$ 18.8$ billion generated in 2007. Based on the latest NPD's

Corresponding Author: Tse Guan Tan, Evolutionary Computing Laboratory,

School of Engineering and Information Technology, Universiti Malaysia Sabah, Malaysia 
Am. J. of Economics and Business Administration 3 (1): 186-190, 2011

Table 1: NPD sales figures for the US computer and video game industry between year 2002 and year 2009

\begin{tabular}{llll}
\hline Year & $\begin{array}{l}\text { Video game } \\
\text { billion (us) }\end{array}$ & $\begin{array}{l}\text { Computer game } \\
\text { billion (us) }\end{array}$ & $\begin{array}{l}\text { Total sales } \\
\text { billion (US) }\end{array}$ \\
\hline 2009 & 19.66 & 0.54 & 20.20 \\
2008 & 21.40 & 0.70 & 22.10 \\
2007 & 17.94 & 0.91 & 18.85 \\
2006 & 12.50 & 0.97 & 13.47 \\
2005 & 10.50 & 0.95 & 11.45 \\
2004 & 9.90 & 1.10 & 11.00 \\
2003 & 10.00 & 1.20 & 11.20 \\
2002 & 10.30 & 1.40 & 11.70 \\
\hline
\end{tabular}

report, the sales of computer and video game industry showed signs of slowing down in 2009 with the revenues of $\$ 20.20$ billion (Riley, 2010). The US game market declines almost 9\% when compared with the same period last year.

Game AI is used to refer to the any type of AI method such as learning, pathfinding, planning and scripting for creation of autonomous Non-Player Characters (NPCs) in computer and video games (Funge, 2004); (Ahlquist and Novak, 2008). That is, the NPCs are able to play the games more intelligently or at a level appropriate to human abilities. In recent years, there has been an increasing interest in game AI. A series of international game AI competitions are organized by highly respected conferences such as IEEE Congress on Evolutionary Computation (CEC), IEEE Conference on Computational Intelligence and Games (CIG) and Conference on Artificial Intelligence and Interactive Digital Entertainment (AIIDE) to encourage academic researches and game developers to investigate and to share the knowledge, experiences, opinions, information as well from each other in this promising area in order to enhance the performance of the NPCs. Additionally, academic research in game AI can bring the benefit to the computer and video games (Forbus and Laird, 2002). Several commercial game companies have the collaboration with universities or research groups for doing the research in this area, for examples Neural Networks Research Group at the Department of Computer Sciences at the University of Texas is working with Digital Media Collaboratory of the IC2 Institute on the AI for Neuro-Evolving Robotic Operatives (NERO, 2003). Another project is the collaboration of The University of Alberta GAMES group and Electronic Arts in FIFA soccer game.

In this study, the Artificial Neural Network (ANN) (Sarhan, 2009; Mehmood et al., 2010; Obe and Shangodoyin, 2010) is evolved with the Evolution Strategies (ES) for the computer player to automatically learn and optimally play the game of Ms. Pac-man. The proposed algorithm will be referred to as an Evolution Strategies Neural Network or ESNet throughout the study. The ESNet is benchmarked against the Random Direction (RandDir) and Random Neural Network (RandNet) in the same domain. The experiments also investigate how the mutation probabilities impact the performance of ESNet. The importance of the proposed algorithm for decision-making in a dynamic environment is that the agent will not only be able to make an intelligent decision like a human player in the computer or video game, but also that the successful application of these techniques will be highly beneficial to the real-world problems, such as in the application of robotics and other complex systems.

The organization of this study is as follows. In Section 2, the experimental materials and methods are illustrated. Subsequently, the benchmarking results and discussions are given in Section 3 and Section 4. Finally, conclusions are shown in Section 5.

\section{MATERIALS AND METHODS}

In this research, the feed-forward ANN (Haykin, 2009) with one hidden layer is applied. The architecture can be briefly described as 5-20-1, with 5 inputs, 20 hidden units and 1 output unit. A log-sigmoid activation function is used for network units. The number of evaluations per run is fixed at 500 in the ESNet, whereas the RandDir and RandNet are evaluated only once game in a run since both algorithms did not involve any learning process. The general parameters are summarized in Table 2. Below we give a description of the three algorithms, RandDir, RandNet and ESNet. The Ms. Pac-man game is used as an application domain to assess the performance of the proposed algorithms.

Random direction (RandDir): RandDir is a very simple controller, which directs the Ms. Pac-man agent to move in random directions in the maze. There are 4 directions available to the agent: up, down, left and right. At each time step or every few time steps, the algorithm applies a random change to the agent's direction.

Random neural network (RandNet): The structure of the RandNet controller is shown in Fig. 1. This system begins with a randomly initialized vector of weights and biases for the ANN from a uniform distribution in the range between -1 and 1, whose output is then used to control the Ms. Pac-man agent. 


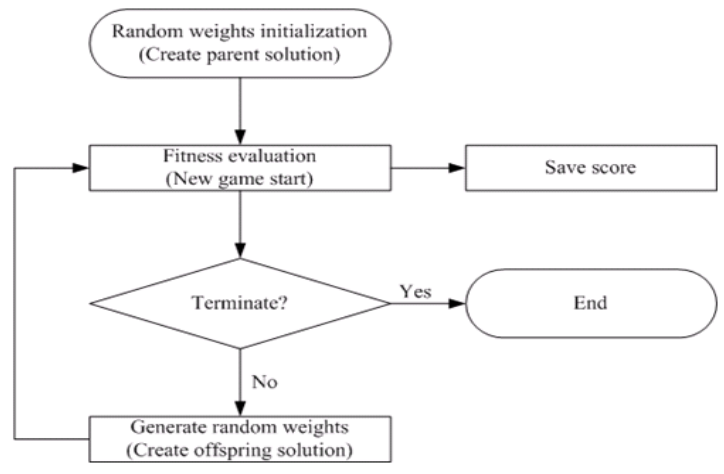

Fig. 1: The flowchart of RandNet algorithm

Table 2: Parameters setting

\begin{tabular}{ll}
\hline Parameters & Setting \\
\hline Number of inputs & 5 \\
Number of outputs & 1 \\
Number of hidden layers & 1 \\
Number of hidden neurons & 20 \\
Activation function & Log-sigmoid \\
Mutation operator & Polynomial mutation \\
Mutation probability & $0.1,0.3,0.5$ and 0.9 \\
Distribution index & 20.0 \\
Number of runs & 10 \\
\hline
\end{tabular}

Evolution Strategies Neural Network (ESNet): ES is a simple and fast algorithm was envisaged by Rechenberg and Schwefel in 1965 as a numerical optimization technique. The (1+1)-ES for a two membered ES has been applied to train the ANN by evolving the weights and biases, called ESNet. In the initialization phase, the ANN weights and biases are encoded into a chromosome from uniform distribution with range $[-1,1]$ to act as parent and evaluate its fitness. Subsequently, polynomial mutation operator was used to create an offspring from the parent and evaluate its fitness. After that, the offspring and parent are compared. If the offspring performs better than the parent, then the parent is replaced by the offspring as new parent for the next evaluation (generation). Otherwise the offspring is eliminated and a new mutated offspring is generated. If parent and offspring are incomparable, the offspring is compared with set of previously nondominated individuals in the archive. Fig. 2 shows the flowchart of ESNet.

The fitness function $\mathrm{F}$ chosen for maximization is based on the score obtained in each evaluation as follows:

$\mathrm{F}=\sum_{\mathrm{n}=1}^{\mathrm{N}}$ (Ms. Pacman Scores)

where, $n$ and $N$ represent the number of lives in a full game.

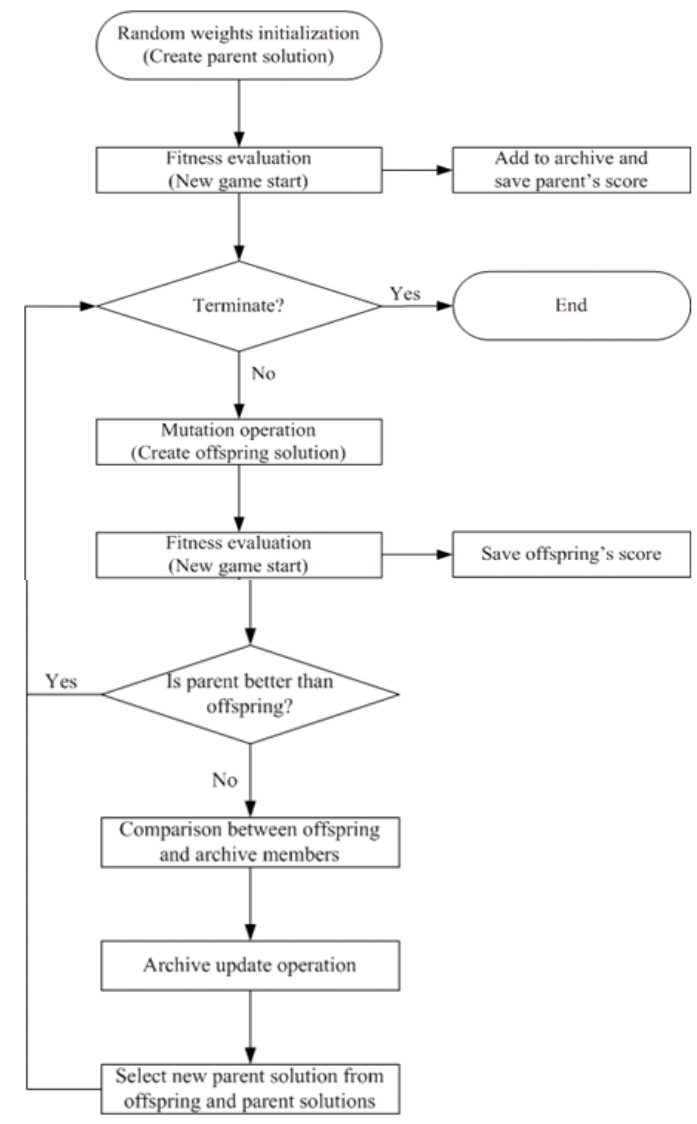

Fig. 2: The flowchart of ESNet algorithm

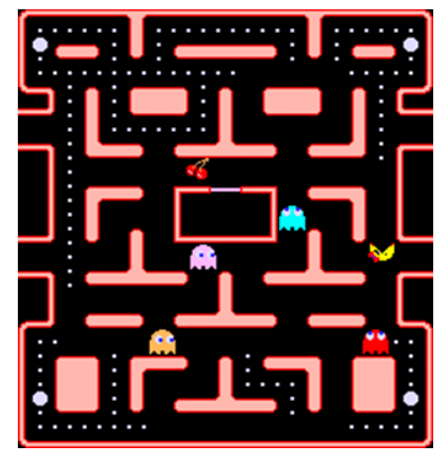

Fig. 3: A snapshot of the Ms. Pac-man game

Ms. Pac-man: Ms. Pac-man is a famous maze-based game from Midway Manufacturing in 1981 as shown in Fig. 3. The objective of Ms. Pac-man is simply to survive as long as possible, while achieving the highest possible score. Ms. Pac-man was inherited from Pacman, therefore the basic game-play of Ms. Pac-man still mostly remains the same as the original game. The agent (yellow circle with a ribbon) is controlled by the 
Am. J. of Economics and Business Administration 3 (1): 186-190, 2011

player to be moved around the maze in any of the four directions (up, down, left or right) attempting to eat the pills and fruits and to evade the ghosts. There are four ghosts named Blinky (red colored), Pinky (pink colored), Inky (blue colored) and Sue (orange colored) in the game that chase the agent and try to kill it in the maze. The maze is filled with hundreds of pills (small dots) and four power pills (large dots. When the agent eats a power pill, the ghosts will change to become edible ghosts (dark blue colored), so that the agent can then chase and gobble down the ghosts to earn some extra points for a limited period of time. Another good way to collect extra points is by eating the fruits that appear twice in every level that bounce around the maze. The first fruit appears after agent has eaten 64 pills (including power pills) whereas the second fruit appears after agent has eaten 176 pills. By default, Ms. Pac-man initially has three lives, however, if the total score reaches 10000 points, an extra life will be awarded, while the player loses a life when a ghost kills the agent. The game will proceed to the next level when all the pills on the maze had been eaten. As the levels increase, the degree of speed and difficulty increase as well, thus making the game more challenging. The game is considered ended when the player loses all lives or completes all levels.

\section{RESULTS}

Table 3 presents the experimental data for 10 independent runs to compare the performances of RandDir, RandNet and ESNet. There were significant differences in the scores for ESNet with mutation probability $0.9($ Mean $=6175)$, RandNet $($ Mean $=557)$ and RandDir (Mean = 439). In addition, we studied the effects of different mutation probabilities. A series of experiments were conducted with the mutation probabilities 0.1, 0.3, 0.5 and 0.9 respectively in ESNet. Table 4 shows overall the experimental data for ESNet. As can be seen from the table, ESNet with mutation probability 0.9 obtained significantly better outcomes than the other three mutation probabilities, 0.1 (Mean = 2503), $0.3($ Mean $=5543)$ and $0.5($ Mean $=6000)$.

\section{DISCUSSION}

According to the analyzed results, the performance of ESNet is noticeably better than that of RandDir, RandNet and ESNet with lower mutation probabilities. It seems that as the mutation probabilities increased, the performance of ESNet increased as well. Furthermore,
Table 3: Ms. Pac-man obtained scores

\begin{tabular}{llll}
\hline Run & RandDir & RandNet & $\begin{array}{l}\text { ESNet (Mutation } \\
\text { Probability =0.9) }\end{array}$ \\
\hline 1 & 450 & 700 & 6350 \\
2 & 410 & 720 & 5700 \\
3 & 470 & 670 & 6680 \\
4 & 430 & 260 & 5920 \\
5 & 420 & 200 & 6020 \\
6 & 460 & 650 & 6270 \\
7 & 420 & 420 & 5960 \\
8 & 470 & 560 & 6420 \\
9 & 460 & 900 & 6500 \\
10 & 400 & 490 & 5930 \\
Mean & 439 & 557 & 6175 \\
\hline
\end{tabular}

Table 4: ESNet with different mutation probabilities

\begin{tabular}{lllll}
\hline & Mutation Probability & & \\
Run & - & & \\
\hline 1 & 0.1 & 0.3 & 0.5 & 0.9 \\
2 & 2860 & 5910 & 6250 & 6350 \\
3 & 2050 & 4530 & 6430 & 5700 \\
4 & 2950 & 5670 & 5310 & 6680 \\
5 & 2430 & 5280 & 5560 & 5920 \\
6 & 1760 & 4980 & 5570 & 6020 \\
7 & 2890 & 5900 & 6040 & 6270 \\
8 & 2910 & 5990 & 6080 & 5960 \\
9 & 1600 & 5100 & 6030 & 6420 \\
10 & 2360 & 5920 & 6620 & 6500 \\
Mean & 3220 & 6150 & 6110 & 5930 \\
\hline
\end{tabular}

it was shown to produce relatively better results compared with other previous studies that utilized a computational intelligence approach (Lucas, 2005; Handa, 2010; DeLooze and Viner, 2009). Hence, we have shown empirical evidence that ESNet can improve the learning capability of the ANN by evolving their weights and biases in a dynamic video game setting.

\section{CONCLUSION}

ESNet is introduced in this study to create an intelligent Ms. Pac-Man agent to play the game. This proposed algorithm has been tested against RandDir and RandNet and the results revealed that the ESNet clearly outperforms both random systems. Overall, ESNet has been successfully used to optimize the performance of ANN, which has proven that an automatic game AI generation system can be realized through the hybridization of evolutionary algorithms and artificial neural networks for the video game industry.

\section{ACKNOWLEDGMENT}

This research is funded under the ScienceFund project SCF52-ICT-3/2008 granted by the Ministry of Science, Technology and Innovation, Malaysia. 
Am. J. of Economics and Business Administration 3 (1): 186-190, 2011

\section{REFERENCES}

Ahlquist, J.B. and J. Novak, 2008. Game Development Essentials: Game Artificial Intelligence. 1st Edn., Cengage Learning, Asia, ISBN-10: 1-4180-3857-1, pp: 271.

DeLooze, L.L. and W.R. Viner, 2009. Fuzzy q-learning in a nondeterministic environment: Developing an intelligent ms. pac-man agent. Proceeding of the IEEE Symposium on Computational Intelligence and Games, Sept. 7-10, Milano, Italy, pp: 162-169. DOI: 10.1109/CIG.2009.5286478

Forbus, K.D. and J. Laird, 2002. Ai and the entertainment industry. IEEE Intell. Syst., 17: 15-16. DOI: 10.1109/MIS.2002.1024746

Funge, J.D., 2004. Artificial Intelligence for Computer Games: An Introduction. 1st Edn., AK Peters, Australia, ISBN: 9781568812083, pp: 160.

Handa, H., 2010. Constitution of Ms.Pacman player with critical-situation learning mechanism. Int. J. Know. Eng. Soft Data Paradigms, 2: 237-250. DOI: 10.1504/IJKESDP.2010.035906

Haykin, S., 2009. Neural Networks and Learning Machines. 3rd Edn., Prentice Hall, USA., ISBN10: 0-13-147139-2, pp: 936.

Jayakanthan, R., 2002. Application of computer games in the field of education. Elect. Library, 20: 98-102. DOI: 10.1108/02640470210697471

Lucas, S.M., 2005. Evolving a neural network location evaluator to play Ms.Pac-Man. Proceeding of the IEEE Symposium on Computational Intelligence and Games, Apr. 4-6, Colchester, United Kingdom, pp: 203-210.

Mehmood, H., N.K. Tripathi and T. Tipdecho, 2010. Indoor positioning system using artificial neural network. J. Comput. Sci., 6: 1219-1225. DOI: 10.3844/jcssp.2010.1219.1225

NERO, 2003. Neuro-Evolving Robotic Operatives. WareSeeker.com.

http://linux.wareseeker.com/Games/neuroevolving-robotic-operatives-1.01.zip/339401
NPD, 1967. NPD Group. NPD Group, Inc., http://www.npd.com/corpServlet?nextpage=corp_ welcome.html

Obe, O.O. and D.K. Shangodoyin, 2010. Artificial neural network based model for forecasting sugar cane production. J. Comput. Sci., 6: 439-445. DOI: 10.3844/jcssp.2010.439.445

Riley, D.M., 2004. The NPD Group Reports Annual 2003 U.S. Video Game Industry Driven by Console Software Sales. NPD Press. http://www.npd.com/press/releases/press_040126a.htm

Riley, D.M., 2005. The NPD Group Reports Annual 2004 U.S. Video Game Industry Retail Sales. NPD Press. http://www.npd.com/press/releases/press_050119.html

Riley, D.M., 2006. The NPD Group Reports Annual 2005 U.S. Video Game Industry Retail Sales. NPD Press. http://www.npd.com/press/releases/press_060117.html

Riley, D.M., 2007. 2006 U.S. Video Game and PC Game Retail Sales Reach \$13.5 Billion Exceeding Previous Record Set in 2002 by Over \$1.7 Billion. NPD Press. http://www.npd.com/press/releases/press_070119.html

Riley, D.M., 2008. 2007 U.S. Video Game and PC Game Sales Exceed \$18.8 Billion Marking Third Consecutive Year of Record-Breaking Sales. NPD Press. http://www.npd.com/press/releases/press_080131b.html

Riley, D.M., 2010. 2009 U.S. Video Game Industry and PC Game Software Retail Sales Reach \$20.2 Billion. NPD Press. http://www.npd.com/press/releases/press_100114.html

Sarhan, A.M., 2009. Iris recognition using discrete cosine transform and artificial neural networks. J. f Comput. Sci., 5: 369-373. DOI: 10.3844/jcssp.2009.369.373 